\title{
A Survey of Automatic Text Summarization System for Different Regional Language in India
}

\author{
Virat V. Giri, Dr.M.M. Math and Dr.U.P. Kulkarni
}

\begin{abstract}
Automatic text summarization is technique of compressing the original text into shorter form which will provide same meaning and information as provided by original text. The brief summary produced by summarization system allows readers to quickly and easily understand the content of original documents without having to read each individual document. The overall motive of text summarization is to convey the meaning of text by using less number of words and sentences. Summaries are of two types: Abstractive summaries and Extractive summaries. Extractive summaries involve extracting relevant sentences from the source text in proper order. The relevant sentences are extracted by applying statistical and language dependent features to the input text. On the other hand, abstractive text summaries are made by applying natural language understanding. This system comprises of two main steps: Pre Processing and Processing phase. Pre Processing phase represents the Marathi text in structured way. In processing phase, different features deciding the importance of sentences are determined and calculated. Some of the statistical features are Marathi keywords identification, relative sentence length feature and numbered data feature. Various linguistic features for selecting important sentences in summary are: Marathi headlines identification, identification of lines just next to headlines, identification of Marathi-nouns, identification of Marathi-proper-nouns, identification of common-EnglishMarathi-nouns, identification of Marathi-cue-phrases and identification of title-keywords in sentences. Scores of sentences are determined from sentence-feature-weight equation. Weights of features are determined using mathematical regression. This paper concentrates on survey and performance analysis of automatic text summarizers for Marathi language.
\end{abstract}

Keywords--- Marathi Text Summarizer, Extractive Summarization, Named Entity Recognition, Keywords Identification, Headlines Identification.

\section{INTRODUCTION}

Automatic text summarization [1] deals with reducing the source-text into a shorter version preserving its contents and overall meaning. Generally there are two phases of text

Virat V. Giri, Research Scolar, KLS, Gogte Institute of Technology, Udyambag, Belgaum. E-mail:virat.giri@gmail.com

Dr.M.M. Math, Professor, Department of Computer Science and Engg, KLS, Gogte Institute of Technology, Udyambag, Belgaum. E-mail:mmmath@git.edu

Dr.U.P. Kulkarni, SDM College of Engineering and Technology, Dharwad.E-mail:upkulkarni@yahoo.com

DOI:10.9756/BIJSESC.8242 summarization systems: 1) Pre- Processing-phase represents the source text in structured way. 2) In Processing phase different features deciding the importance of sentence are determined and calculated. Scores of sentences are determined using equation of feature weights and high scored sentences in proper order as of input text are extracted for final summary. This paper describes single document multi news Marathi extractive summarizer. It is text extraction based summarization system which is used to summarize the single Marathi document with multi news by retaining the relevant sentences based on statistical and linguistic text features. Maharashtra is one of Indian states and Marathi is its official language. For Marathi language, it is the only summarizer available as no other Marathi summarizer exists. This summarizer has two phases. 1) Pre-processing phase includes finding boundary of Marathi sentences, Elimination of Marathi-stop-words, Stemmer for Marathi nouns and proper names, allowing input restrictions to input text, Elimination of duplicate sentences and normalization of Marathi noun words in noun morph. 2) In processing phase, different features deciding the importance of sentences are determined and calculated. Some of the statistical features are Marathi keywords identification, relative sentence length feature and numbered data feature. Various linguistic features for selecting important sentences in summary are: Marathi headlines identification, identification of lines just next to headlines, identification of Marathi-nouns, identification of Marathi-proper-nouns, identification of common- English Marathi-nouns, identification of Marathi-cuephrases and identification of title-keywords in sentences etc. Sentencefeature-weight equation is applied for finding the final-scores of sentences. Weights of each feature are calculated using weight learning methods. Top ranked sentences in proper order are selected for final summary at selective compression ratios.

\section{BACKGROUND}

\section{A. Text Summarizers for Indian Languages}

Various automatic text summarization systems are commercially or non-commercially available for most of the commonly used natural languages. Most of these text summarization systems are for English and other foreign languages. Moreover, for commercial products the technical documentation is often minimal or even absent. When it comes to Indian languages, automatic text summarization systems are still lacking. Various text summarizers for Indian languages are discussed below:

\section{B. Bengali Language}

a) Islam and Masum (2004) developed corpus oriented text summarization system 'Bhasa' for Bengali language. It 
is based on scoring the files of corpus in which query words are having highest frequency and then producing the summary of text documents on the basis of query words by applying vector-space-term-weighting. Tokenizer is able to determine different terms, abbreviations, tags and boundary of sentences and to denote terms, headings, titles and sentences using markups by semantic and syntactic analysis [2].

b) Das and Bandyopadhyay (2010) developed Bengaliopinion text summarizer based on given topic which can determine the information on sentiments in the input text. Then this information is aggregated for denoting text summary. It applies a model on topicsentiment for determination and aggregation of sentiments. It is implemented for theme determination at the discourse level. Moreover aggregation is performed by clustering of theme using k-means approach and by applying the megraph representation at relational level which is ultimately applied for selection of relevant sentences in summary by using page rank standard approach.[3]

c) Sarkar (2012) proposed Bengali text summarization by sentence extraction and has investigated the impact of thematic term feature and position feature on Bengali text summarization. The proposed summarization method is extraction based. It has three major steps: (1) preprocessing (2) sentence ranking (3) summary generation. The pre-processing step includes stop-word removal, stemming and breaking the input document in to a collection of sentences. After an input document is formatted and stemmed, the document is broken into a collection of sentences and the sentences are ranked based on two important features: thematic term and position. It proposed another approach for summarizing Bengali news documents. It describes system that produces extractive summaries of Bengali news documents.[4]

\section{Tamil language}

a) Kumar and Devi (2011) proposed Tamil languagesummarization system for scoring of sentences insummary using graph theoretic scoring technique. Thissystem uses statistics of frequency of words and a termpositional and weight-age calculation by string pattern for scoring of sentences.[5]

b) Banu et al. (2007) proposed text summarizer for Tamil documents using technique of semantic graph by identifying Subject Object Predicate from individual lines for making semantic-graph of source text document and its corresponding summary generated by human experts.[6]

c) Banu (2010) proposed another technique for summarizing documents of Tamil by using approach of sub graph for selecting lines from source document treated as text summary or another technique for generating a generic summary of document. In this system, syntax of language neutral, which is the system for representing the natural language lines, has been applied for compressing the text documents. [6]

\section{Kannada Language}

a) Kallimani et al. (2010) proposed a text summarizer for Kannada i.e. "AutoSum” a named IR system using Text Summarization of some Regional Language in India. This system processes the input text and then decides which lines are relevant and which lines are not relevant. User interaction in this system is command based interaction. In it, text is summarized on console. The output summary of this system can be produced either in simple text or in hypertext mark up language. If hypertext mark-up language is used in output then relevant lines are highlighted.[8]

b) Jayashree et al. (2011) proposed a text summarization system for Kannada named "Kannada text Summarizer based on Key terms Extraction”. This system takes pre classified Kannada documents obtained from online web resources and identifies the thematic words from these documents by mixing GSS (Galavotti, Sebastiani, Simi) coefficients and Inverse-Document-Frequency techniques with Term-Frequency and then apply these extracted keywords for making summary.[9]

c) Jayashree et al. (2012) proposed another pre-classified documents summarizer for Kannada by scoring of sentences which retrieves key terms from Kannada documents, by combining GSS (Galavotti, Sebastiani, Simi) coefficients and Inverse-Document-Frequency techniques with Term Frequency for retrieving key term and then applies them for summarizing the text. Kannada text files are taken from Webdunia. Webdunia is a special web portal in Kannada that is used for latest News, Entertainment News, Sports related news, Jokes and Shopping etc.[10]

d) Kallimani et al. (2012) proposed a new technique for summarizing the longer text documents by considering one of the South Indian regional languages (Kannada). It deals with a single document summarization based on statistical approach. The purpose of summary of an article is to facilitate the quick and accurate identification of the topic of the published document. The objective is to save prospective readers' time and effort in finding the useful information in a given huge article.[11]

\section{E. Punjabi Language}

a) Gupta et al. (2012) proposed of Punjabi text summarizer. It makes extractive summary for Punjabi text by extracting the important lines based on language oriented features and features belonging to statistics oftext. Every line of input text is treated as vector of different features like sentence relative length, Punjabicue terms, Punjabi terms belonging to nouns, terms belonging to common nouns of English and Punjabi, Punjabi named entities, location of lines, Term-Frequency and Inverse-SentenceFrequency scores for extracting thematic terms, existence of numeric data in lines etc. Duplicate sentences are eliminated in the preprocessing phase. Weight-age of sentence-features which are influencing the different lines is calculated by applying regression which is a weight learning method.[7] [12][13]. 


\section{F. Hindi Language}

a) Garain et al. (2006) proposed text summarization software for Hindi text has been commercially developed by CDAC (Centre for development of advance computing) Noida. This system has applied statistical approach; Language based approach along with heuristic approach for developing text summarization system for Hindi. This summarization software includes 1) Features based on Statistics: Term, Pair of Terms, Particular Cue terms, count, determining Value of Threshold, location of sentences and proper location scheme etc. 2) Analysing language oriented features: Determine noun terms, terms existing together, finding stop-words, terms which are functional in nature. 3) Language oriented Psycho features: Unique or duplicate terms. 4) Feature belonging to Heuristics: sentence belonging to Title, Location, Number of words in a sentence and Table of contents etc. 5) Giving weightage, ranking of lines etc. [15]

G. Text pictures for Indian Language

a) Garain et al. (2006) proposed text summarization of compressed text pictures for Indian language. This system is used to summarize JBIG2 coded text pictures without using optical character recognition. Compressed pictures are decompressed and then sentences and terms are marked.[15]

\section{H. Multi-Language}

a) Keyan (2012) proposed multi-lingual (Tamil and English) multi-document summarization by neural networks. The system involves three steps. In first step, the sentences of the documents are converted into vector form. In the second step weight values are assigned to vector form based on sentence features. Depend on sentence weight value, single document summarization is done. The output of single document summarization is used as an input for multi-document Summarization. Final step is a sentence selection, in which output summary is selected based on the similarity and dissimilarity measures. Sentence similarity and dissimilarity measures are used to compare the sentences. From that, resultant summary is produced. The proposed system can be able to summarize both Tamil and English online newspapers.[13]

b) Patel et al. (2007) proposed a technique to text summarization for English, Hindi, Gujarati and Urdu documents. The algorithm is based on structural and statistical (rather than semantic) factors. The algorithm has been applied on document understanding conference (DUC) data English documents and various newspaper articles for other languages with corresponding stop words list and modified stemmer.[14]

\section{PERFORMANCE COMPARISON IN INDIAN SUMMARIZERS}

Following table shows the comparison of performance of some of existing summarizers for Indian languages.
Table 1: Performance of Some of Existing Summarizers for Indian Languages

\begin{tabular}{|c|c|c|}
\hline \multirow[t]{2}{*}{ Summarization systems } & \multicolumn{2}{|c|}{ Performance comparison of existing } \\
\hline & $\operatorname{Accuracy}(\operatorname{In} \%)$ & Test used \\
\hline $\begin{array}{l}\text { Punjabi Text } \\
\text { Summarization System }\end{array}$ & $\begin{array}{l}\text { For Stories: } 89.32 \% \\
\text { (At 30\% Compression } \\
\text { Ratio) } \\
\text { For News } \\
\text { Documents:95.32\% } \\
\text { (At 30\% Compression } \\
\text { Ratio) }\end{array}$ & F Score \\
\hline $\begin{array}{l}\text { Bengali Summarizer } \\
\text { using Textual Images }\end{array}$ & $56 \%$ & Efficiency \\
\hline $\begin{array}{l}\text { Bengali Summarizer } \\
\text { using Text Extraction }\end{array}$ & $\begin{array}{l}84 \% \\
\text { (At } 40 \% \text { Compression } \\
\text { Ratio) }\end{array}$ & Efficiency \\
\hline $\begin{array}{l}\text { Topic based Bengali } \\
\text { Opinion Summarizer }\end{array}$ & $69.65 \%$ & Efficiency \\
\hline $\begin{array}{l}\text { Multi Lingual Summarizer } \\
\text { for English, Hindi, } \\
\text { Gujarati } \\
\text { \& Urdu }\end{array}$ & $82 \%$ & Efficiency \\
\hline $\begin{array}{l}\text { Document Summarizer } \\
\text { for Kannada }\end{array}$ & $\begin{array}{l}\text { For Literature: } 70 \% \\
\text { For Entertainment: } 80 \% \\
\text { For Sports: } 76 \%\end{array}$ & Efficiency \\
\hline $\begin{array}{l}\text { Summarization from } \\
\text { large Kannada documents } \\
\text { using a novel approach }\end{array}$ & $\begin{array}{l}\text { At } 30 \% \text { Compression } \\
\text { ratio: } 80 \% \\
\text { At } 40 \% \text { Compression } \\
\text { Ratio: } 83.33 \%\end{array}$ & Efficiency \\
\hline $\begin{array}{l}\text { Tamil text extraction } \\
\text { system for an } \\
\text { agglutinative language }\end{array}$ & Score $: 0.47$ & ROUGE - 1 \\
\hline
\end{tabular}

\section{STEPS FOR TEXT SUMMARIZATION FOR MARATHI} LANGUAGE

1. To implement text extraction based Marathi text summarization system for single stories, single news documents and multi news documents.

2. To implement stemmer for Marathi nouns and proper nouns by making different kinds of stemming rules for Marathi language.

3. To implement rule based Marathi named entity recognition system for recognizing Marathi proper names in source text.

4. To implement TF-ISF based keywords identification system for Marathi language.

5. To implement other features for Marathi text summarization and feature weight learning using mathematical regression.

6. To assemble these tools and develop a complete automatic text summarization system for Marathi language that will summarize the Marathi text based on compression ratio given.

7. To evaluate the summary based on combination of both intrinsic and extrinsic summary evaluation techniques for Marathi language.

\section{PROPOSED SUMMARIZATION METHOD}

The proposed summarization method is extraction based. It has three major steps: 


\section{[1] Pre-processing (2) Sentence ranking 3) Summary generation.}

A. Preprocessing

The pre-processing step includes stop-word removal, stemming and breaking the input document in to a collection of sentences. For stop word removal, we have used the Marathi top-word list downloadable from the website of Forum for Information Retrieval Evaluation.

\section{B. Stemming}

Using stemming, a word is split into its stem and affix. The design of a stemmer is language specific, and requires some significant linguistic expertise in the language. A typical simple stemmer algorithm involves removing suffixes using a list of frequent suffixes, while a more complex one would use morphological knowledge to derive a stem from the words.

\section{Sentence Ranking}

After an input document is formatted and stemmed, the document is broken into a collection of sentences and the sentences are ranked based on two important features: thematic term and position.

\section{Pre-Processing Phase of Marathi TeXt SUMMARIZATION}

A. Marathi words and Sentences boundary identification - From the Marathi text, remove the punctuation mark characters like; . ““”: - -- space character, tab space and so on for finding individual Marathi words. In Marathi language sentence boundary is identified by presence of vertical bar I, question mark?, Exclamation sign!, Enter key, new line character etc at the end of sentence.

B. Marathi Language Stop Word Elimination - Marathi language Stop words are frequently occurring words in Marathi text. Eliminate these words from text; otherwise, sentences containing them can get influence unnecessarily. Some commonly occurring Marathi stop words.

C. Marathi Language Noun and Proper Name Stemming - The purpose of stemming is to obtain the stem or radix of those words which are not found in dictionary/morph.

D. It is first time that Marathi stemmer for nouns and proper names has been developed and implemented by us. Various steps for Marathi stemmer are as follows:-

1. Each word of Marathi text is checked against Marathi noun morph and proper nouns list.

2. If word is found in Marathi noun morph or Marathi proper name list, its corresponding score is incremented by 1 .

3. If word is not found in Marathi noun morph or Marathi proper name list then Stemming is performed using any of stemming rules.

4. The stemmed words are again checked against Marathi noun morph or proper names list for possibility of Marathi noun or proper name.

5. The accuracy percentage of correct words detected under various rules of Stemmer
A. Applying input restrictions to input text - Marathi Text Summarization system allows Unicode based Gurmukhi text as input and can accept maximum up to 1,00000 characters as input otherwise it will give error message. Moreover majority of characters should be in Gurmukhi.

B. Normalization of Marathi nouns - many of the popular Marathi noun words are written in multiple ways. To overcome this problem, input Marathi text and Marathi noun morph has been normalized for different spelling variations of Marathi noun words.

C. Elimination of Duplicate Sentences - In Marathi Text Summarization system, duplicate sentences is deleted from input by searching the current sentence in to the sentence list which is initially empty. If current sentence is found in sentence list then that sentence is set to null otherwise it is added to the sentence list being the unique sentence. This elimination prevents duplicate sentences from appearing in final summary.

\section{Processing Phase of MARATHI TeXt SUMMARIZER}

A. Marathi sentence relative length - Very short sentences are avoided for including in final summary as often they contain less information. On the other hand lengthy Marathi sentences might contain lot of information. This feature is calculated by dividing number of words in a sentence with word count of largest sentence. Its value will be always less than or equal to one.

B. Marathi keywords identification - It is first time that Marathi keywords identification system is developed and implemented by us. Keywords are helpful in deciding the sentence importance. Marathi Keywords are identified by calculating TF-ISF (Term FrequencyInverse Sentence Frequency) calculation.

C. Number Feature - Numeric data is very important. Numeric data includes numbers and digits like 3, 45 and 80 etc. The sentence that contains numeric data is important and it is most probably included in the document summary. Numeric data also includes Gurmukhi and Roman numerals. The score for this feature is calculated as the ratio of the number of numeric data in sentence over the sentence length.

D. Headline/Next line identification-In single/multi news documents, headlines are most important and are always included in the final summary. Line just next to headline might contain very important information related to summary and is usually included in summary. In Marathi headlines detection system, if current sentence does not ends with punctuation marks like 'I ' vertical bar, exclamation sign '!' and question mark '?' etc. but ends with enter key or new line character then set the headline flag for that line to true. If the next subsequent line of this headline ends with punctuation marks like 'I' vertical bar, exclamation sign '!' and question mark '?' etc. but does not ends with enter key or new line character then set the next line flag to true for that line. 
E. Marathi noun feature - Those Marathi sentences containing nouns are important. Input words are checked in Marathi noun morph for possibility of nouns. Marathi noun feature score is calculated by dividing number of Marathi nouns in a sentence with length of that sentence. The value of this feature for a sentence will be from 0 to 1 .

F. Common English-Marathinoun feature - Majority of such terms are not found in Marathi dictionary or Marathi nounmorph. and sentences containing them are important. Common English-Marathi noun feature score is calculated by dividing number of common English-Marathinouns in a sentence with length of that sentence.

G. Marathi proper names feature - Proper names are the names of person, place and concept etc. not occurring in dictionary. Marathi Named Entity Recognition is also used for identifying Marathi proper names. Marathiproper name feature score is calculated by dividing number of Marathiproper names in a sentence with length of that sentence.

H. Marathi named entity recognition - It is first time that rule based Marathi named entity recognition system has been developed and implemented by us. Prior to it, no other rule based Marathi named entity system was available. It uses various gazetteer lists like prefix list, suffix list, middle name list, last name list and proper name lists for checking whether the given word is proper name or not.

I. Marathi cue phrase feature - Cue Phrases are certain keywords like In conclusion, Summary and Finally etc. These are very much helpful in deciding sentence importance. Those sentences which are beginning with cue phrases or which contain these cue phrases are generally more important than others. Firstly a list of Marathi Cue phrases has been made and then those sentences containing these Cue phrases are given more importance.

J. Marathi title keywords feature- Title lines are the headlines of single/multi news documents. Those Punjabi sentences containing title keywords are important.

\section{CONCLUSION}

Most of the lexical resources used in pre-processing and processing such as Marathi stemmer, Marathi proper name list, English-Marathi noun list, Marathi keywords extraction, Marathi rule based named entity recognition etc. had to be developed from scratch as no work had been done in that direction.

1. Intrinsic and Extrinsic measures are to be used for summary evaluation.

2. For Marathi news documents, Marathi Text Summarization system performs very well compression ratio.

\section{REFERENCES}

[1] V. Gupta and G.S. Lehal, "A survey of text summarization extractive techniques", Journal of emerging technologies in web intelligence, Vol. 2, No.3, Pp.258-268, 2010.

[2] T. Islam and S.M.A. Masum, "Bhasa: A Corpus Based Information Retrieval and Summarizer for Bengali Text", Macquarie University, Sydney, Australia, 2004.

[3] A. Das and S. Bandyopadhyay, "Topic-based Bengali opinion summarization", In Proceedings of the 23rd International Conference on Computational Linguistics: Posters, Pp. 232-240, 2010.

[4] K. Sarkar, "An approach to summarizing Bengali news documents". In proceedings of the International Conference on Advances in Computing, Communications and Informatics, Pp. 857-862, 2012.

[5] S. Kumar, V.S. Ram and S.L. Devi, "Text Extraction for an Agglutinative Language", Proceedings of Journal: Language in India, Pp. 56-59, 2011.

[6] M. Banu, C. Karthika, P. Sudarmani and T.V. Geetha, "Tamil Document Summarization Using Semantic Graph Method", Proceedings of International Conference on Computational Intelligence and Multimedia Applications, Vol. 2, Pp. 128-134, 2007.

[7] V. Gupta and G.S. Lehal, "Automatic Text Summarization for Punjabi Language", International Journal of EmergingTechnologies in Web Intelligence, Vol. 5, No. 3, Pp. 257-271, 2013.

[8] J.S. Kallimani, K.G. Srinivasa and B. R. Eswara,"Information Retrieval by Text Summarization for an Indian Regional Language," In Proceedings ofInternational Conference on Natural Language Processing and Knowledge Engineering, pp. 1-4, 2010.

[9] R. Jayashree, K.M. Srikanta and K. Sunny, "Document Summarization in Kannada using Keyword Extraction", Proceedings of AIAA 2011,CS\& IT 03, Pp. 121-127, 2011.

[10] R. Jayashree, "Categorized Text Document Summarization in the Kannada Language by Sentence Ranking", Proceedings of 12th International Conference on Intelligent Systems Design and Applications (ISDA), Pp. 776-781, 2012.

[11] J. S. Kallimani, K.G. Srinivasa and B.R. Eswara, "Summarizing News Paper Articles: Experiments with Ontology Based, Customized, Extractive Text Summary and Word Scoring", Journal of Cybernetics and Information Technologies, Bulgarian Academy of Sciences, Vol. 12, No. 2, Pp. 34-50, 2012.

[12] Gupta and G.S. Lehal, "Complete Preprocessing Phase of Punjabi Language Text Summarization", International Conference on Computational Linguistics COLING'12, IIT Bombay, India, Pp. 199205, 2012.

[13] M.K. Keyan and K.G. Srinivasagan, "Multi-Document and MultiLingual Summarization using Neural Networks", Proceedings of International Conference on Recent Trends in Computational Methods, Communication and Controls,Pp. 11-14, Vol. 5, 2012.

[14] A. Patel, T. Siddiqui and U.S. Tiwary, "A language independent approach to multilingual text summarization", Proceedings of conference RIAO ‘07, Pittsburgh PA, U.S.A., Pp. 123-132, 2007.

[15] U. Garain, A. K. Datta, U Bhattacharya and S.K. Parui, "Summarization of JBIG2 Compressed Indian Textual Images," Proceeding of 18th International Conference onPattern Recognition (ICPR'06), IEEE, Kolkata, India, Vol. 3, Pp. 344-347, 2006.

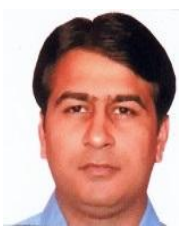

Virat V. Giri completed his graduation in Information Technology from Dr. BANU Aurangabad in 2004 andPost-graduation from Bharathi Vidyapeeth Pune in 2011. He started his carrier as lecturer from JSPM Pune. In JSPM Pune He has worked in different capacity as lecturer, HOD and In charge Principal. He joined Sanjay Ghodawat Polytechnic in 2012 as Principal. Currently is in doing his research under the guidance of Dr Math M M from VTU Belgavi. $\mathrm{He}$ is Life member if ISTE.He has published 5 papers in international journal and 4 papers in international conference.

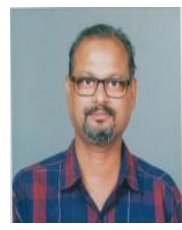

Dr.M.M. Math, received his Master's degree (M.S) from Birla Institute of Technology, Pilani, and Rajasthan.He has completed his PhD degree under Graphics Era University, Deharadun. Research Center: -SDMCET, Dharwad, Karnataka under the guidance of Prof. Umakant $P$. Kulkarni. He is currently working as Associate Professor in Information Science Department, Gogte Institute of 
Technology, Belgaum, and Karnataka. He has presented and published papers at IEEE, Springer, Elsevier andnational andInternational conferences. His research interests include Autonomic computing, Pervasive computing and their applications and visual sensor network

Dr.U.P. Kulkarni received his $\mathrm{PhD}$ degree from Shivaji University, Kolhapur. Research Center Walchand College of Engineering \& Tech, Sangli, Maharashtra in Nov 2007. He has completed his Master's degree (M.E) from PSG, Coimbatore. He is currently working as Professor in Computer Science Department, SDMCET, Dharwad, and Karnataka. He has published and presented a number of papers in many reputed journals and at International IEEE conferences as well. He also has presented technical talks and tutorials on various aspects of Mobile Agents. His research interests include mobile agents, computer networking, and distributed networking 